Medical and psychological

\author{
clinic \\ "Family plus"
}

\title{
Effect of perinatal stress on the reproductive system.
}

\author{
Pechnikova E. Yu.
}

XVI European psychology Congress

02-05 July 2019

Moscow 
- In today's world, full of various stressors, the first place go psychological stressors - emotional and information, as well as environmental.

- In the new man-made conditions, the health of the next generations, which are still laid in utero, remains important. Stress is a modifying factor and an element of evolutionary selection. However, the term "prenatal stress" (Ward, $1972,1984)$, which appeared in the 70 s of the $X X$ century, was filled with new content in the XXI century. 
- Unlike an adult, whose hormonal homeostasis in the hypotolamo-pituitary-adrenocortical system is carried out on the principle of negative feedback between the adrenal corticosteroids and the hypothalamic-pituitary system, in the fetus at the end of the gestational period, glucocorticoids stimulate the secretion of $\mathrm{ACTH}$, i.e., act on the mechanism of positive feedback. Therefore, the stress of the mother's body causes a complex of neurohormonal changes in the fetus, which can be qualified as a stress state of the fetus.

- Perinatal stress causes a complex of various morphological (micro-and ultrastructural), neurochemical, endocrine, metabolic changes that are fixed in postnatal life. 
- As a result of hormone-neurotransmitter imprinting of the brain, a certain type of nerve connections, the level of excitability of neurons, their susceptibility to the activating action of the same hormones and mediators, which in early ontogenesis served as inducers of cell differentiation, are formed.

- Demonstration of pathological manifestations of disorders of sexual differentiation of the brain (SDB - androgen-depended formation of morphological and functional sex differences in the structures of the Central nervous system). 
- Changes in the phenotype of neuroendocrine structures are localized mainly in the preoptic region, where the center of regulation of male sexual behavior is located. Increasing the level of corticosteroids in the blood of the mother and fetus, as well as reducing the concentration of norepinephrine in the hypothalamus of the fetus significantly violates SDB, with the most vulnerable male fetus, which is associated with a high risk of brain demasculization. In adults, homosexual behavior, violation of the regulation of gonadotropin secretion and at the same time - a pronounced weakening of the ability of the system "pituitaryadrenal cortex" to adequately respond to stress stimuli can be detected. 
- Women whose mothers have experienced various stressful situations during pregnancy are more likely to have reproductive disorders, primarily in terms of fertility decline. When taking pregnant hormonal drugs for medical reasons progestins with weak androgenic activity or synthetic estrogen - , as well as during pregnancy in women with an excess of male sex hormones of different Genesis, changes in sexual behavior in female descendants and sexual orientation reversion are possible. 


\section{Adaptive fetal response}




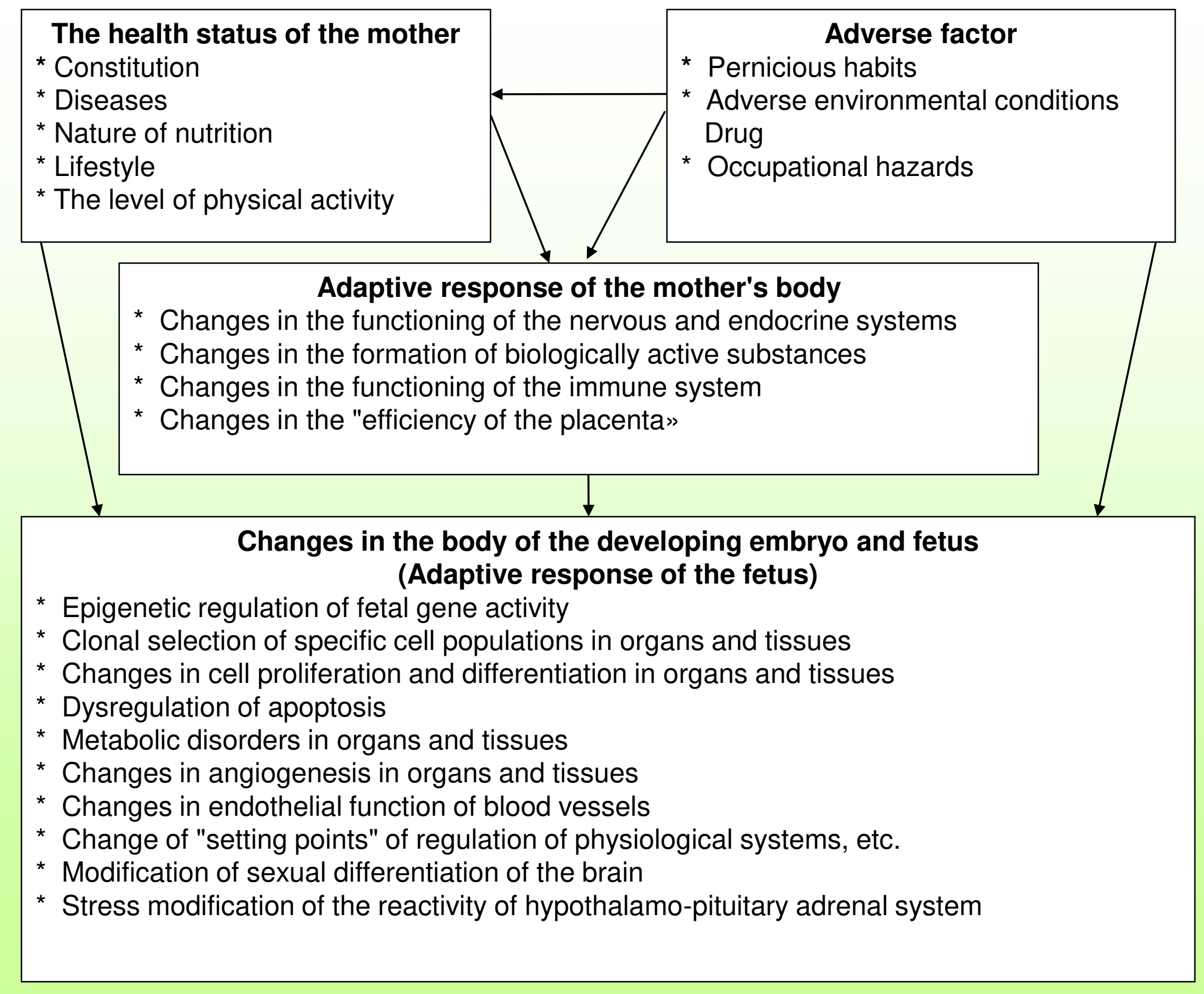




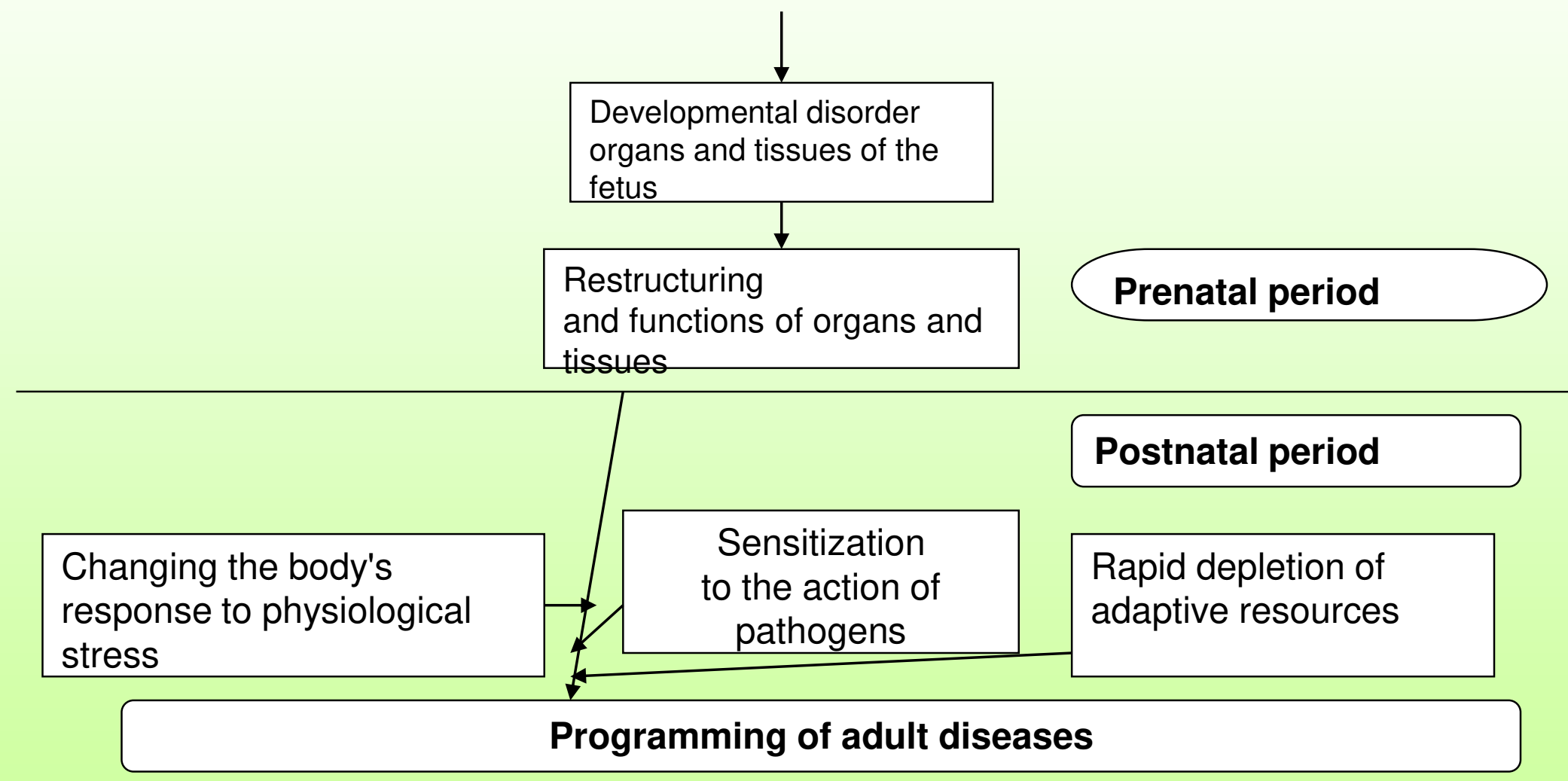


- In both sexes, prenatal stress leads to reduced stress resistance and rapid depletion. Violations of the subsequent stages of ontogenesis are superimposed on the formed consequences of perinatal stress and lead to a wide range of violations in the psychological and physiological sphere of men and women. 
- It is necessary to prevent perinatal stress in order to preserve the health of future generations:

- further development of ideas about perinatal stress and methods of its diagnosis;

- informing and educating the population about preventive measures. 